\title{
Multiple Embolism in a Female Patient with Infective Endocarditis. Low Back Pain and Hematuria as the Initial Clinical Manifestations
}

\author{
Marcelo Luiz Campos Vieira, Mônica Luisa Rappi Schmidt, Marcos Valério Coimbra de Resende, \\ Luis Sérgio Afonso de André Júnior
}

São Paulo, SP - Brazil

\begin{abstract}
A 59-year-old female patient with mitral valve prolapse and a previous history of lumbosacral spondyloarthrosis and lumbar disk hernia had an episode of infective endocarditis due to Streptococcus viridans, which evolved with peripheral embolism to the left kidney, spleen, and left iliac artery, and intraventricular cerebral hemorrhage. Her clinical manifestations were low back pain and hematuria, which were initially attributed to an osteoarticular condition. Infective endocarditis is a severe polymorphic disease with multiple clinical manifestations and it should always be included in the differential diagnosis by clinicians.
\end{abstract}

Infective endocarditis remains a challenge for clinical diagnosis. It may have a wide range of initial manifestations and should be included in the clinical differential diagnosis. Despite advances in echocardiographic investigation, directed antibiotic therapy, and early surgical treatment, infective endocarditis remains an important cause of mortality among the infectious diseases. Therefore, its early identification is fundamental for reducing its complications and mortality. We report a case of infective endocarditis in a female patient with mitral valve prolapse, whose initial clinical presentation was low back pain, hematuria, and low fever.

\section{Case report}

A 59-year-old white female patient was admitted to the emergency sector of our hospital complaining of low back pain, hematuria, and mild fever for 2 days. She reported un-

Hospital e Maternidade São Luiz - São Paulo

Mailing address: Marcelo Luiz Campos Vieira - Rua Cardoso de Melo, 463/21 04548-002 - São Paulo, SP, Brazil - E-mail: mlcvieira@aol.com English version by Stela Maris C. e Gandour dergoing a dental procedure 30 days earlier. On physical examination, the patient was in good general condition, eupneic, afebrile, and with healthy coloring. Her systemic arterial blood pressure was $130 / 70 \mathrm{mmHg}$, her heart rate was $80 \mathrm{bpm}$, and her respiration rate was $18 \mathrm{bpm}$. Her cardiovascular, pulmonary, and neurological examinations were within the normal range. On urinary sediment examination, hematuria was detected with no leukocyturia and proteinuria. The patient was medicated for her clinical complaints and was advised to return for ambulatory follow-up.

The patient returned to the emergency service 4 weeks after her first visit, reporting at that time persistent low fever for 2 weeks. On physical examination, she was in good general condition, dyspneic, her mucosas were pale $(+/ 4+)$, and no petechiae could be seen. Her systemic arterial blood pressure was $100 / 60 \mathrm{mmHg}$, her heart rate was $110 \mathrm{bpm}$, and her axillary temperature was $38.4^{\circ} \mathrm{C}$. On cardiac auscultation, a $3^{\text {rd }}$ cardiac sound (B3) and a systolic murmur $(+++/ 4+)$ in the mitral area with irradiation to the left axillary region could be heard. The examination of the abdomen showed a mass in the left hypochondrium, and the examination of the lungs revealed crepitant rales in both bases. The neurological examination was within the normal range. The electrocardiogram revealed sinus rhythm, with the cardiac axis at $60^{\circ}$, and an overload pattern in the left chambers. The chest $X$-rays showed enlargement of the cardiac silhouette $(++/ 4+)$ due to left ventricular enlargement. Her hemogram results were as follows: hemoglobin of 8.4 (11.5-16.0) g/dL; leukocytes of $13,200(4,500-10,000) / \mathrm{mm}^{3}$, with $10(3-5) \%$ band neutrophils, and 75 (54-62)\% segmented neutrophils; $13(20-35) \%$ lymphocytes; 2 (3-8)\% monocytes; and 130,000 (150,000$400,000) / \mathrm{mm}^{3}$ platelets. The blood sedimentation rate was 30 (20-40) $\mathrm{mm} / \mathrm{h}$; sodium of $138(136-145) \mathrm{mEq} / \mathrm{L}$; potassium of $4.3(3.6-5.4) \mathrm{mEq} / \mathrm{L}$; urea of $34(15-45) \mathrm{mg} / \mathrm{dL}$; and creatinine of $1.0(0.9-1.4) \mathrm{mg} / \mathrm{dL}$. The urinary sediment examination showed mild hematuria. On abdominal computed tomography, the spleen was enlarged, had an irregular and bossela- 
ted contour and a peripheral septate hypodense area in its inferior pole, which was not highlighted after injection of contrast medium. The left kidney had normal dimensions and regular contours, and a hypodense area of well-defined contours in its inferior pole, which was not highlighted after contrast medium injection. These hypodense areas represent splenic and renal infarcts. The computed tomography of the lumbosacral vertebral column showed diffuse osteopenia, irregularities of the vertebral bodies determined by osteophyte projections, lumbosacral spondyloarthrosis, right centrolateral intervertebral disk hernia from $\mathrm{L}_{4}$ to $\mathrm{L}_{5}$, and protrusion of the $\mathrm{L}_{3}-\mathrm{L}_{4}$ and $\mathrm{L}_{5}-\mathrm{S}_{1}$ disks. On transthoracic echocardiography, the left ventricular measures were $5.7 \times 3.8$ $\mathrm{cm}$, the left ventricular ejection fraction was 0.71 , and the left atrial diameter was $5.2 \mathrm{~cm}$. The morphological analysis revealed significant mitral valve prolapse of the anterior and posterior leaflets, with an image suggestive of vegetation attached to the posterior leaflet, and significant valvular regurgitation. The transesophageal echocardiography confirmed the presence of the image of a pediculate highly mobile vegetation of homogeneous echotexture, measuring more than $10 \mathrm{~mm}$ in its larger diameter, and adhered to the atrial portion of the posterior leaflet of the mitral valve (fig. 1). Blood cultures were positive for Streptococcus viridans, but the species was not determined.

The patient received intravenous antibiotic therapy, which initially consisted of the association of ceftriaxone and amikacin, later replaced by the association of vancomycin and amikacin. Cardiac surgery was indicated due to refractory heart failure and was performed on the $3^{\text {rd }}$ day of antibiotic therapy. The mitral valve was replaced by a porcine biological prosthesis, and the presence of vegetation on the posterior leaflet of the mitral valve was confirmed. A small atrial septal defect of the ostium secundum type was identified and repaired. Neither abscesses nor cavitary fistulas were found.

On the $2^{\text {nd }}$ postoperative day, the patient experienced a sudden episode of significant pain in the left lower limb, accompanied by the absence of perception of pulses in the

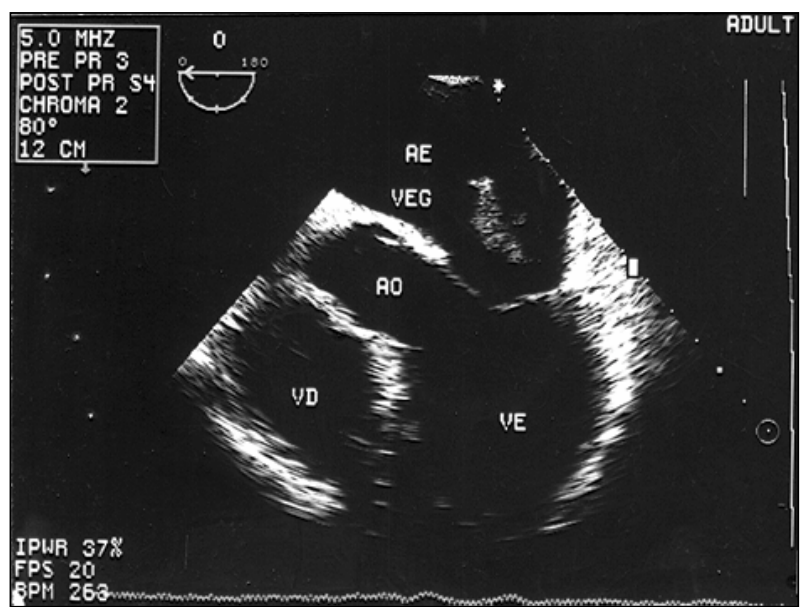

Fig. 1 - Transesophageal echocardiogram. Image at $0^{\circ}$ revealing a bizarre vegetation in the mitral valve. left femoral, popliteal, and tibial arteries. Aortic arteriography was performed and showed occlusion of the left iliac artery (fig. 2). The thrombus was removed through the passage of a 5F Fogarty catheter. On clinical evolution, the patient had ischemia in 4 of her left toes, which partially improved. A slight elevation in urea and creatinine levels was observed with a later return to normal values.

On the $20^{\text {th }}$ postoperative day, the patient experienced mental confusion, psychomotor agitation, and right hemiparesis. The computed tomography of the skull revealed intraventricular hemorrhage with significant ventricular flooding, and weakening of the cerebral sulci and cisterns (fig. 3). The patient required mechanical ventilatory support and intense in-hospital physical therapy. She was discharged after 104 days of hospitalization with right hemiparesis and dysarthria.

\section{Discussion}

Infective endocarditis remains a severe disease with significant mortality and morbidity rates. In a series of 300

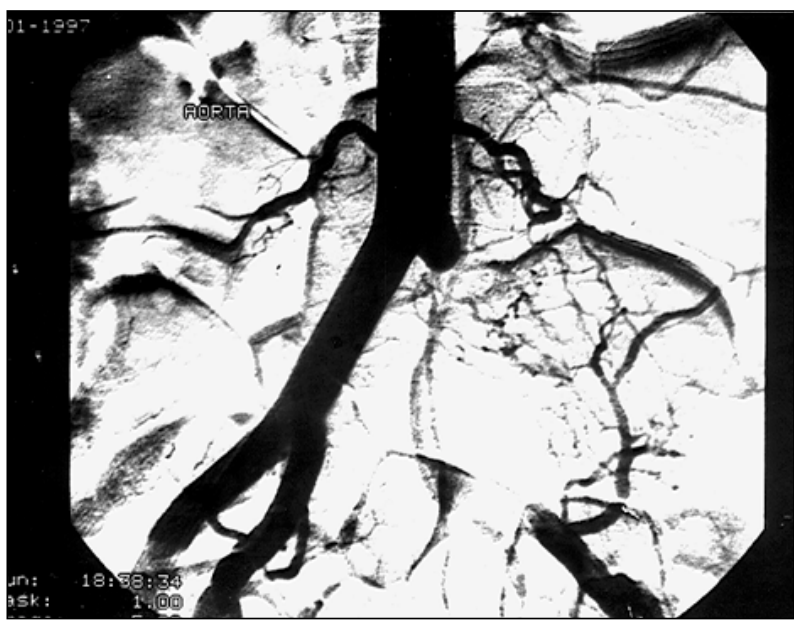

Fig. 2 - Aortic angiography. Note occlusion of the left iliac artery.

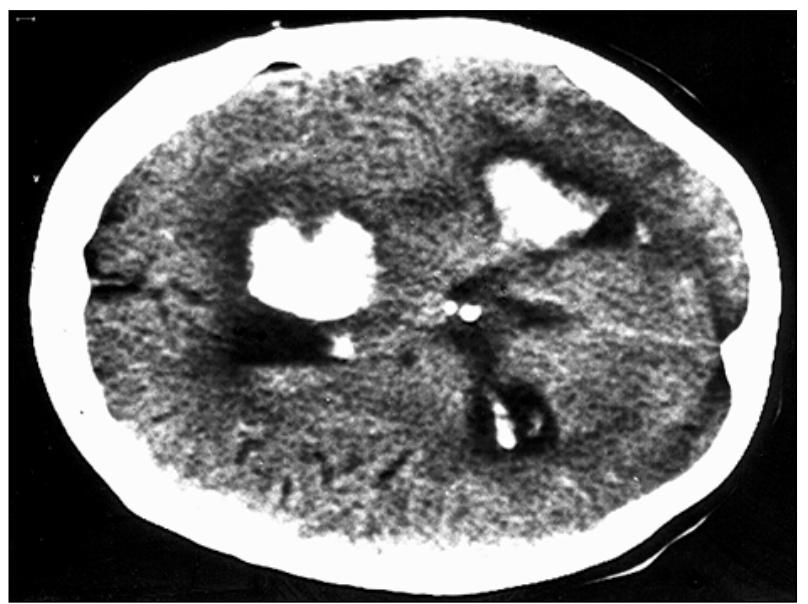

Fig. 3 - Computed tomography of the skull. Cerebral hemorrhage with intense ventricular flooding. 
episodes of infective endocarditis in 287 patients at a Brazilian referral hospital ${ }^{1}, 389$ clinical complications were observed, 72 of which were of a neurological nature; splenic abscesses or infarcts were reported in only 3 cases. Peripheral septic embolism is an extremely prevalent phenomenon in infective endocarditis, being present in approximately $45 \%$ to $65 \%$ of the cases studied on autopsy ${ }^{2}$. Peripheral septic embolism is not always clinically identified because of the lack of diagnostic investigation. This is more specifically the case of renal and splenic septic embolism, in which the diagnostic investigation of low back or abdominal pain is not routinely performed. Usually, renal embolism accompanied by renal infarct may be suggested by a history of low back pain, fever, transitory micro- or macrohematuria, and leukocytosis, accompanied by progressive renal failure. In $25 \%$ of the cases, renal embolism may occur with no exuberant symptoms and be identified only on anatomicopathological examination ${ }^{3}$.

Peripheral embolism is more related to infective endocarditis of the left chambers (more prevalent in infective endocarditis of the mitral valve than in that of the aortic valve) in the presence of highly mobile large vegetations ${ }^{4,5}$. The highest indices of peripheral embolism occur when the vegetations are adhered to the mitral anterior leaflet; when the vegetations are located in the posterior leaflet, as in our patient, the indices are lower ${ }^{6}$. In up to $65 \%$ of the patients with septic embolism, involvement of the central nervous system occurs. Septic embolism to vasa vasorum or to the intraluminal space with infection of the intima layer may lead to the formation of mycotic aneurysms, which may rupture and bleed. The occurrence of neurological events negatively influences the prognosis of infective endocarditis, with an up to 2.3 times higher mortality ${ }^{7}$.

We report a case of infective endocarditis caused by Streptococcus viridans in a patient with no previous known history of cardiovascular disease. Our patient ignored the fact that she had mitral valve prolapse with valvular insufficiency. She underwent dental manipulation with no previous prophylactic antibiotic therapy and evolved to septic embolism to multiple organs. The clinical findings were initially attributed to low back pain resulting from osteomuscular disease (lumbosacral spondyloarthrosis and disk hernia). Low back pain is known to be a very common symptom, and its etiological diagnosis is extensive and difficult. Low back pain may be the initial clinical presentation of infective endocarditis and should be part of the differential diagnosis in patients who have low back pain of sudden onset, a history of fever, and cardiac auscultation suggestive of valvular heart disease.

Likewise, hematuria has a wide differential diagnosis. The possibility of renal infarct should be considered in patients with valvular heart disease, atrial fibrillation, or suspected infective endocarditis. Usually, the rate of embolism decreases until the end of the second week of antibiotic therapy. In the case reported, the patient also had an episode of embolism to the central nervous system 23 days after beginning antibiotic therapy. The cerebral ictal episode may have resulted from the infective process. We cannot disregard the patient's age and the presence of previous heart disease in the etiological identification of the cerebral event.

The identification of larger vegetations is usually related to infective endocarditis caused by staphylococci, fungi, or Haemophilus. In the present case, we observed vegetation of significant dimensions, which is not usually found in endocarditis caused by streptococci.

Another significant aspect of this case is the need for ambulatory diagnosis of valvular heart diseases so that previous antibiotic prophylaxis may be recommended in dental procedures for these patients.

\section{References}

1. Mansur AJ, Grimberg M, Da Luz PL, Bellotti, G. The complications of infective endocarditis: a repraisal in the 1980s. Arch Intern Med 1998; 152: 2428-32.

2. Nakayama DK, $\mathrm{O}$ 'Neill JA, Wagner H. Management of vascular complications of bacterial endocarditis. J Pediatr Surg 1986; 21: 636-41.

3. Dorrler J, Wahba A. Diagnosis and treatment of visceral and renal embolisms. Herz 1991; 16: 425-33.

4. Fowler VG, Sanders LL, Kong LK, et al. Infective endocarditis due to Staphylococcus aureus: 59 prospectively identified cases with follow-up. Clin Infect Dis 1999; 28: 106-14.
5. Heinle S, Wilderman N, Harrison K, et al. Value of transthoracic echocardiography in predicting embolic events in active infective endocarditis. Am J Cardiol 1994; 74: 799-801.

6. Rohmann S, Erbel R, Gorge G, et al. Clinical relevance of vegetations localization by transesophageal echocardiography in infective endocarditis. Eur Heart J 1992; 13: 446-52.

7. Lunardi W, Grimberg M, Scaff M, et al. Neurological events in infective endocarditis. Arq Bras Cardiol 1993; 61: 349-55. 